\title{
Qualitative Exploration of a Client-Centered, Goal-Directed Approach to Community-Based Occupational Therapy for Adults With Traumatic Brain Injury
}

\author{
Emmah Doig, Jennifer Fleming, Petrea L. Cornwell, Pim Kuipers
}

\section{KEY WORDS}

- brain injuries

- community health services

- goals

- patient participation

- rehabilitation
Emmah Doig is PhD Candidate, School of Health and Rehabilitation Sciences, The University of Queensland, Brisbane, Queensland 4062 Australia; emmah.doig@optusnet.com.au

Jennifer Fleming, PhD, is Senior Research Fellow in Occupational Therapy, School of Health and Rehabilitation Sciences, The University of Queensland and the Princess Alexandra Hospital, Brisbane.

Petrea L. Cornwell, PhD, is Research Fellow in Speech Pathology, School of Health and Rehabilitation Sciences, The University of Queensland and the Princess Alexandra Hospital, Brisbane.

Pim Kuipers, MA, Grad Dip Rehab, PhD, is Senior Research Fellow, Centre for Remote Health, Joint Centre of Flinders University and Charles Darwin University, Alice Springs, Northern Territory, Australia.
OBJECTIVES. We explored the clinical application of goal-directed therapy in community-based rehabilitation from the perspective of clients with traumatic brain injury (TBI), their significant others, and their treating occupational therapists.

METHOD. Twelve people with TBI and their significant others completed an outpatient, goal-directed, 12week occupational therapy program. Semistructured interviews with 12 participants, 10 significant others, and 3 occupational therapists involved in delivering the therapy programs explored their experiences of goal-directed therapy.

RESULTS. Participants, their significant others, and therapists described goal-directed therapy positively, expressing satisfaction with progress made.

CONCLUSION. Goals provide structure, which facilitates participation in rehabilitation despite the presence of barriers, including reduced motivation and impaired self-awareness. A therapist-facilitated, structured, goalsetting process in which the client, therapist, and significant others work in partnership can enhance the process of goal setting and goal-directed rehabilitation in a community rehabilitation context.

Doig, E., Fleming, J., Cornwell, P. L., \& Kuipers, P. (2009). Qualitative exploration of a client-centered, goal-directed approach to community-based occupational therapy for adults with traumatic brain injury. American Journal of Occupational Therapy, 64, 559-568.

oal setting has been described as "the essence of rehabilitation" (Barnes \& Ward, Q 2000, p. 8) and the "cornerstone of effective rehabilitation" (Lawler, Dowswell, Hearn, Forster, \& Young, 1999, p. 402). Goals facilitate a client-centered approach to rehabilitation because they can be uniquely adapted to each person according to his or her profile of strengths and limitations (Malec, 1999). Goals also provide a concrete and meaningful way of measuring progress in rehabilitation, and goal achievement has been used extensively to evaluate the effectiveness of programs (Joyce, Rockwood, \& Mate-Kole, 1994; Lannin, 2003; Ottenbacher \& Cusick, 1989; Trombly, Radomski, Trexel, \& Burnet-Smith, 2002). Moreover, it is widely recognized that the use of goals that are important and meaningful to the client can have a motivating effect in terms of encouraging participation in rehabilitation (Malec, 1999; Pollock, 1993; Shut \& Stam, 1994). Occupational therapists use client-centered approaches to provide meaningful, purposeful goals that empower the client to actively participate in and improve their occupational performance (Pedretti, 1996). Goal-directed therapy is dependent on the implementation of a goal-planning process that identifies client-centered goals.

Previous quantitative investigations and case studies have shown that the use of client-centered goal planning with adults undergoing neurological rehabilitation can improve self-perceived and observed goal performance and satisfaction 
(Bodiam, 1999; Eames, Ward, \& Siddons, 1999; Gagne \& Hoppes, 2003; Jenkinson, Ownsworth, \& Shum, 2007; Phipps \& Richardson, 2007; Trombly, Radomski, \& Davis, 1998; Trombly et al., 2002; Wilson, Evans, \& Keohane, 2002). Direct involvement in individualized goal planning has been shown to result in better maintenance of treatment gains for clients with traumatic brain injury (TBI) than for a group with lesser involvement in goal planning (Webb \& Glueckauf, 1994). A client-centered approach involves goals that are set by the client on the basis of his or her own definition of the problems; the approach enables greater selfdetermination and control and enhances the person's potential for active participation (Pollock, 1993). Because occupational performance encompasses activities in the areas of self-care, productivity, and leisure and is influenced by environmental and societal factors (Reed \& Sanderson, 1980 ), it is unique to each person and requires individualized measurement tools sensitive to varying needs and situations (Pollock, 1993).

Client-centered rehabilitation has been described by people with long-term physical disabilities as individualization of programs to meet their needs, including preparation for life in the real world, mutual participation with health professionals in decision making and goal planning, meaningful outcomes, and family involvement throughout the rehabilitation process (Cott, 2004). Because of the established efficacy of client-centered approaches, this study used a client-centered, goal-planning approach to guide community-based occupational therapy programs for a group of people with severe TBI who were recently discharged from inpatient brain injury rehabilitation.

Challenges to achieving a client-centered, goal-directed approach to the rehabilitation of people with TBI have been widely documented and include cognitive impairment, reduced motivation, and diminished self-awareness. Typically, people with TBI demonstrate reduced self-awareness and tend to underreport impairments (Sherer et al., 1998; Toglia \& Kirk, 2000); they therefore may have difficulty identifying realistic therapy goals (Fleming \& Strong, 1995), may not realize the need for therapeutic intervention and, consequently, demonstrate poorer outcomes (Fischer, Gauggel, \& Trexler, 2004). Moreover, goal setting requires client participation in the process, and passivity of clients has been identified as a barrier to goal setting (Seigert \& Taylor, 2004). Cognitive and motivational factors associated with TBI may further affect the client's ability to engage in goal planning, thus potentially limiting its usefulness with this population (Fleming \& Strong, 1995). Research investigating the impact of brain injury-related changes on client participation in goal planning is limited (Fischer et al., 2004; Kuipers, Foster, Carlson, \& Moy, 2004).
Multiple factors that are unique to the community rehabilitation context need to be considered when applying a goal-directed rehabilitation approach. The goals of people with brain injury after discharge from the hospital become broader and more complex in nature (Seigert \& Taylor, 2004) and move from a focus on physical rehabilitation toward a more varied focus with greater emphasis on reintegration over time (Kuipers et al., 2004). Accordingly, the context of community rehabilitation will affect the dynamic of goal setting. Most research on goal setting, however, has occurred in inpatient settings. A study that explored approaches to goal setting from the perspective of therapists working in an inpatient geriatric rehabilitation unit found that therapist-directed and -led approaches were more commonly described in practice than were client-centered approaches (Leach, Cornwell, Fleming, \& Haines, in press). Another study found that clients in an inpatient geriatric stroke setting showed a lack of participation in the goalsetting process (Wressle, Oberg, \& Henriksson, 1999). In addition, staff members working primarily in inpatient neurological rehabilitation units reported that it was often difficult for clients to set goals in the early stages after onset of significant disability and identified home visits as useful for assisting in identification of clients' needs, roles, and family conflicts (Playford et al., 2000).

These findings contrast with the opinions of therapists working with people with brain injury in community-based rehabilitation settings where the importance of therapists' developing goals with clients rather than imposing goals was emphasized (Kuipers et al., 2004). Once discharged from the hospital into the community, family members are increasingly involved in goal setting (Seigert \& Taylor, 2004). The home setting is more conducive to involving the family in goal setting because of increased access to family members and opportunities to involve the family (Doig, Fleming, \& Kuipers, 2008). Family involvement in goal setting may assist with obtaining a holistic understanding of the client's social support and home situation and identifying goals (Kuipers et al., 2004), and it may be particularly helpful in setting realistic therapy goals when the client has impaired selfawareness and tends to underreport impairments.

Goal-directed approaches in rehabilitation settings have mostly been evaluated using quantitative methodologies. Improvements in goal performance after participation in acquired brain injury (ABI) rehabilitation programs, including people with TBI, have been reported in outpatient clinic rehabilitation settings (Phipps \& Richardson, 2007; Trombly et al., 1998, 2002; Webb \& Glueckauf, 1994) and community-based group programs (Jenkinson et al., 2007). A study by Lannin (2003) reported improvements in goal performance using goal attainment scaling (GAS; Kiresuk \& 
Sherman, 1968) in a community rehabilitation program primarily for people with $\mathrm{ABI}$. These findings indicate the success of goal-directed programs for people with ABI. Some studies of people with TBI, however, have excluded participants with impaired self-awareness because they have been unable to identify problem areas and set goals (Trombly et al., 1998, 2002). Moreover, Seigert and Taylor (2004), in discussing the theoretical aspects of goal planning in rehabilitation, reported not only the need to look at "what works" but also the need to explain "how" an intervention works to guide development of effective techniques.

Although quantitative investigations lend evidence for what works for groups of participants, qualitative investigations enable an in-depth analysis of the impact of multiple intrinsic (e.g., injury factors including impaired selfawareness) and extrinsic (e.g., contextual) factors on the process of goal planning and goal-directed therapy. Such investigations also explain why goal planning and goaldirected therapy may work or fail in specific cases in which multiple, interacting factors affect outcome and subsequently inform advancements in rehabilitation practice. Moreover, given the increased likelihood of involvement of family in community settings, qualitative studies taking into account the perspectives of the multiple parties involved in goal planning and goal-directed rehabilitation provide an opportunity to thoroughly explore these process issues.

Previous qualitative studies that have explored goal planning and goal-directed therapy in neurorehabilitation have primarily focused on groups of people with stroke (Lawler et al., 1999; Leach et al., in press; McAndrew, McDermott, Vitzakovitch, Warunek, \& Holm, 1999; Playford et al., 2000; Wressle, Eeg-Olofsson, Marcusson, \& Henriksson, 2002; Wressle et al., 1999). Of those studies that have described and discussed goal planning and goal-directed rehabilitation for people with $\mathrm{ABI}$, the perspectives and opinions of staff members (i.e., therapists, doctors, or nurses) have been described in isolation (Kuipers et al., 2004; Leach et al., in press; Levack, Dean, McPherson, \& Siegert, 2006; Playford et al., 2000; Sumison, 2004). Only one study investigated goal setting in the community rehabilitation context (Kuipers et al., 2004), and the study was not specific to TBI.

Studies have compared the perspectives of clients with stroke (McAndrew et al., 1999; Wressle et al., 1999) and clients with spinal cord injury (MacLeod \& MacLeod, 1996) with staff perspectives about goal setting and rehabilitation. Findings have demonstrated that perspectives of clients and staff differ on goal planning (MacLeod \& MacLeod, 1996; McAndrew et al., 1999) and thereby highlight the benefit of considering and comparing multiple perspectives. Moreover, most previous studies with $\mathrm{ABI}$ samples have explored the goal-planning process or goal content (Kuipers et al., 2004;
Leach et al., in press; McAndrew et al., 1999; Playford et al., 2000; Wressle et al., 1999) with less exploration of the treatment validity of goals or the impact that goals have on the process of rehabilitation and outcomes (Lawler et al., 1999; Levack et al., 2006; MacLeod \& MacLeod, 1996; Wressle et al., 2002).

Despite the emerging emphasis on client-centeredness and goal setting in the literature, no studies have reported and contrasted the perspectives of clients, significant others, and therapists on goal-directed therapy in a community context. In this study, we used a qualitative approach to examine the experiences of clients, family members, and therapists involved in client-centered, goal-directed therapy; the aim was to gain further insight into the benefits and challenges of this process.

\section{Method}

\section{Research Design}

We used a qualitative approach using semistructured interviews (Mason, 2002) of participants, their nominated significant others, and treating occupational therapist. Participants were 12 people with TBI living in the community who had been recently discharged from an inpatient brain injury rehabilitation program in Brisbane, Queensland, Australia, and 10 of their nominated significant others. All participants were receiving outpatient, clinic-based occupational therapy rehabilitation at a large metropolitan hospital.

Participants were eligible to be included in the study if they (1) were between ages 16 and 65; (2) had recently been discharged from inpatient rehabilitation in the Princess Alexandra Hospital Brain Injury Rehabilitation Unit (BIRU); (3) had a diagnosis of TBI, as evidenced by loss of consciousness, a period of posttraumatic amnesia, or computed tomography scan diagnosis; (4) communicated in English; (5) had a significant other available to participate in the study; (6) were referred for an outpatient occupational therapy program at the BIRU that was anticipated to be of at least 18 weeks' duration; and (7) had given informed consent or guardian consent. Exclusion criteria for the study included (1) the presence of factors that would prevent engagement in community-based rehabilitation on the grounds of risk to the visiting therapist or the participant, (2) low level of arousal, (3) coma, (4) severe persisting confusion, (5) significant diagnosed premorbid psychiatric disorder or neurological injury, and (6) significant drug or alcohol problem in which the person was not actively involved in a program of treatment.

As part of their involvement in this research project, the participants received a 12 -week program of goal-directed 
occupational therapy in addition to their regular outpatient occupational therapy program at the outpatient unit (day hospital). Individualized goals were used to direct the content of the therapy program, which was conducted by three qualified, experienced occupational therapists who were employed by the research team. The therapy program consisted of six therapy sessions in the participant's home environment and six sessions in the day hospital setting. The three therapists were also participants in the interview phase of the study.

\section{Participants}

Participants were 10 men and 2 women ranging in age from 18 to 43 years (mean $[M]=24.7$, standard deviation $[S D]$ = 6.9). The mean initial Glasgow Coma Scale (Teasdale $\&$ Jennett, 1974) score was $6(S D=4.2)$, and the average length of posttraumatic amnesia was 86.6 days $(S D=56.4$, missing $=2$ ). Mean length of stay in acute hospital care was 66.7 days $(S D=40.3)$, and mean length of inpatient rehabilitation was 140.1 days $(S D=117.8)$. On discharge from inpatient rehabilitation, the average Disability Rating Scale (Rappaport, Hall, Hopkins, Belleza, \& Cope, 1982) score was 5.6 (SD = 1.7) and average total FIMTM (Hamilton, Grainger, Sherwin, Zielezny, \& Tashman, 1987) score was 108.9 (range = 95$121, S D=9.4)$. On discharge, the average total Self-Awareness of Deficits interview (Fleming, Strong, \& Ashton, 1996) score was 4.66 (range $=0-9, S D=2.9)$; most participants demonstrated either moderate $(n=5)$ or severe $(n=3)$ impairment of self-awareness. All participants' significant others who were interviewed were parents and resided with the participant. All three occupational therapists involved in the study were female, had been qualified for $>8$ years, and had experience in brain injury rehabilitation ranging from 1.5 to 18 years.

\section{Procedures}

Ethical clearance was obtained from the relevant hospital and university ethics committees before commencement. A client-centered goal-planning process using the Canadian Occupational Performance Measure (COPM; Law et al., 1991) and GAS took place before each participant's involvement in the 12-week occupational therapy program. Two participants (P12 and P13) were not able to identify problem areas and program goals during administration of the COPM. A therapist-directed approach (Leach et al., in press) was necessary in those cases; it involved the participants' agreeing to have their significant others and usual inpatient occupational therapist consult with the principal researcher, Emmah Doig, to assist with identification of problem areas to identify potential goals. The problem areas and potential goals were then negotiated and agreed on by the 2 participants. In total, 44 goal attainment scales were developed for the 12 participants.

Emmah Doig conducted an interview at the conclusion of the 12-week program. The interview explored the experiences and opinions of the participants and their significant others in relation to their involvement in the goal-directed occupational therapy program. A semistructured format was used to ensure consistency across all interviews while allowing participants the freedom to raise issues not directly related to the questions (Britten, 1995). Strategies suggested by Paterson and Scott-Findlay (2002) for use when interviewing people with TBI were adopted. The recommendations include appropriate interview scheduling, flexible interview process, and priority questions asked first (to avoid fatigue and overstimulation and maximize attention) as well as use of the interviewee's words to focus more direct questioning and prompt recall. All interviews were undertaken at the participants' homes. Semistructured interviews were also conducted with the three therapists who undertook the goal-directed therapy programs with the participants. Therapist 1 had completed the therapy program with 6 participants, Therapist 2 with 3 participants, and Therapist 3 with 3 participants at the time of conducting the interviews. The interviews were designed to draw on the interviewee's experience and opinions about delivery of the goaldirected rehabilitation program. All interviews were audiotaped with the consent of the participants and took an average of $30 \mathrm{~min}$ to complete.

\section{Data Analysis}

Interviews were transcribed verbatim, and the interview responses to the questions relating to goal planning and goaldirected therapy were analyzed. Emmah Doig read the transcripts to become familiar with the content and used the basic principles of the framework method to thematically analyze the data, using a non-cross-sectional organization of the data to identify chunks and summarize the content of each set of interviews (Spencer, Ritchie, \& O'Connor, 2003). Later, initial themes were identified and grouped into categories and subcategories for each set of interviews, and salient common themes were summarized and compared within and across the three sets of interviews (Ritchie, Spencer, \& O'Connor, 2003). To ensure quality and rigor, one interview from each group (participant, significant other, and therapist) was coded independently by Doig and a research assistant. The two coders met later to compare codes and develop a categorization framework by consensus. The agreed categorization framework was used to code all transcripts, and throughout the analysis further colleague checks (Patton, 2002) were carried out to ensure consistency between any new codes and categories identified. NVivo qualitative data analysis software 
(Version 2.0; QSR International, Melbourne, Victoria, Australia) was used to assist with organization and synthesis of the data.

\section{Results}

The participants, significant others, and therapists noted that the goal-planning process and goal-directed therapy were positive. Several themes were common to all groups, and some themes were more strongly emphasized by some groups than others.

\section{Themes Common to Participants With TBI, Their Significant Others, and Therapists}

Four themes were common to the three groups interviewed: (1) provision of structure, (2) goals and motivation, (3) goal ownership, and (4) impact of awareness on participation.

The first theme emphasized the importance of goals in providing structure for rehabilitation. Therapists identified that having goals made it "quite clear about what we were wanting to achieve" (Therapist $[\mathrm{T}] 2$ ), made expectations about the desired outcome clear, and directed the content of the therapy. Structuring therapy according to goals was facilitated by the "fact that they were so specific, that really helped" (T1). One therapist asked clients to rate the level of importance of each goal at the beginning of each session and found the practice beneficial because "I always knew every single session they were completely focused and motivated on what we were doing" (T1). This concept of structure was also commonly raised by family members and was exemplified by statements such as "once we had the goals, we knew what we were aiming for" (significant other [SO] of Participant $[\mathrm{P}] 7$ ) and "she would base the therapy sessions around achieving those goals" (SO of P4). This structure provided by the therapist was seen as necessary because "she wasn't expecting him to structure the session ... she structured it, and I don't think that self-directed structure is possible, you know the things that need to be done to achieve these goals and guide them through that" (SO of P4). Participants with TBI identified that goals provided a "focus" (P11) and "helped keep you on track" (P12) and that having goals provided structure beyond the therapy session because "it made me start prioritizing things around in my day ... it sort of made me start to think about those things" (P5).

The second theme concerned the motivational aspects of goals. Having goals helped because "it gave him something to strive for" (SO of P9). Likewise, "it could become pretty boring if you aren't aware that, 'Hey, this is all going to lead toward something"' (SO of P4). Moreover, when talking about his daughter's reaction to achieving a goal, another commented, "If you've mastered this one well, you want to go onto the next one" (SO of P6). P3 described goal achievement as motivating because "it gave you targets to achieve, and once you achieved them you'd go, 'Well dust it up, that was done,' and you know that you can do it and you can continue to do it." T2 described the time one of her clients achieved her first program goal: "When she was able to dry herself independently, I think we both had tears because it was so exciting. ... [S] he made statements like, 'Well now I know I can do this, I can try other things."”

Seven of the participants identified the third theme, goal ownership. The participants made statements such as "it was me" (P1), "you're doing what you want to do" (P12), "they were from me, they were to do with me and they were for me" (P3), and "I really wanted to do them so I would do all the steps" (P11). Satisfaction was related to choosing the goals for P8, who stated,

If you leave it up to the individual to pick goals or things that are essentially problems for them and they are working towards that, they can see the benefit of their improvements, and obviously they're a lot more satisfied with that.

P5 described his experience of the goal-directed program as being "asked what things I would like to improve," which he contrasted with his experience of other therapies, stating, "You don't have your say of what you want to do." Goal ownership from the therapists' perspectives meant that the goals should be "set with the client input" (T2) and that the client should perceive the level of goal achievement as acceptable, whereby "if you just set the goal to two steps forward to what you think they'll achieve, they won't accept it and therefore may not engage in it" (T1).

Moreover, one therapist explained that the therapy tasks should be relevant to the goal. In relation to the longterm goal of returning to driving, T1 explained that "physically moving the gears and moving your foot on the pedals" would be more acceptable to the client than "getting the coordination in your feet better." Family members commonly agreed that it was important that the goals be the person's own because "if you don't like something, you are not going to put your heart into it" (SO of P6) and "I think if you'd set the goals for him and it wasn't really what he thought he needed, he wouldn't have worked as hard at it" (SO of P5). Family members did not always agree with the goals chosen by the participants and saw other priorities, but they mostly acknowledged "that was his goal, it's not mine, so sure" (SO of P8).

The final theme common to all groups was that awareness of problems can change and participation can improve with experience. The SO of P3 stated, "At the beginning, it was just like, you know, I have to do this and I have to 
do that, but now he realizes that he is actually progressing, he is actually doing really well." P7 described realizing that he had a problem in his goal area of note taking after working on it. T1, in describing the impact of providing therapy and giving feedback about performance to her clients, identified that

some of them got a lot of self-awareness and then realized that actually they were only 2 steps [along the way] and ... to get to the 10 steps it was going to take them a lot longer than they expected, so they were more realistic.

\section{Themes Common to Participants With TBI and Family}

Three themes were common to participants and their significant others: (1) challenges, (2) family involvement, and (3) satisfaction and progress.

Some family members raised the concept that initial goal setting was challenging, particularly in terms of needing more time living with the person to get to know his or her problems: "If we'd had him home for a period of time and then we had to set goals, we would have a far better idea of where he was at" (SO of P12); "[I]t was time consuming, and I was thinking, 'I don't know what his goals are, just get better'" (SO of P7). By contrast, other family members were goal oriented and familiar with goal setting, stating not only the importance of goals but also that goals are a part of life: "I mean, that is what we all do in life, you know, you set yourself a goal ... then your life is directed towards attaining that goal" (SO of $\mathrm{P} 4)$.

The family members of the 2 participants who could not identify their own goals explained what factors they felt affected the ability to set goals, including memory impairment and reduced self-awareness. For example, P12's mother stated that "he wasn't understanding what goals meant." Some other family members also felt that their relatives needed assistance with planning goals, stating, "You need someone to talk you through it and, you know, help you understand, to fine tune it down to the important main few things" (SO of P4) and "Sit down and try to talk him through processes, so then it made him think and then he came up with a couple of ideas, and that was a much better approach" (SO of P13).

Three participants stated that initial goal planning was complex. P3 described setting goals as "a bit complicated at the start ... just confusing, what should I want to do," and P8 used "intuition ... I didn't realize how much of a problem all of those things would have been still further down the track." Others, however, felt "it was pretty easy to find the goals because it was pretty easy to see what I was having trouble with" (P7).

Family involvement was the second theme common to participants with TBI and their significant others. Several participants described family members as important for encouragement: "He'd start to notice my walking getting a bit better, my balance ... so yeah, I think it's good to have someone close to you involved" (P10), and "with her pushing me I'd do it, I'd do a better job" (P9). Some participants described family involvement in goal planning and therapy as positive because "mum would see me most often, she's definitely probably the most knowledgeable about my current situation" (P8) and "my sister knows me more than my therapist so she would tell her how I'm improving” (P7). By contrast, P3 felt his mother's involvement in goal planning was not necessary because "she didn't know exactly what I'm thinking at the time about what I find difficult." Several family members talked about their involvement in the goal planning as "representing the family, to be a support" (SO of P7) and as important because family knowledge can assist in goal planning (e.g., "I felt like I had to tell you where he was at as well from my point of view so you could help him" [SO of P9]).

The third and strongest theme common to participants with TBI and their significant others was the concept of satisfaction with progress. All participants stated that they felt having program goals was beneficial or expressed satisfaction with progress made on their goals. For example, P6 described her goal achievement by saying it made her feel "good, yeah ecstatic, over the moon." Goals were described as "specific goals" (P4), necessary in that "I didn't want to do others that I don't really need to do" (P4), and achievable as "I could do it" (P1) and "by the end of it, those goals were accomplished" (P3). Family members also expressed their satisfaction with their relatives' progress in their goals and generally agreed that the use of goals to direct therapy was a beneficial process.

\section{Themes Common to Therapists and Family}

Three themes were commonly raised by therapists and significant others: (1) cognitive function, (2) goal evolution, and (3) priorities.

In relation to cognitive function, several family members described how memory impairment affected recall of goals. For example, the mother of P12 explained that her son had difficulty understanding the purpose of therapy and engaging in therapy, as evidenced by statements such as, "I still don't see why they are making me do these things." Moreover, two family members emphasized that poor self-awareness affected goal planning "given that he thinks his ability to do those things is fine and 'why should I do them?" (SO of P12) and "he didn't have that insight into his injury or the depth of it or the problems he was having in a lot of areas" (SO of P13).

Several cases described by the therapists highlighted how goals could be used as tools to motivate and improve 
participation in therapy despite cognitive impairment. T2 described working with clients with memory and communication difficulties: "In general, I think they needed more guiding from me to come back to, well, these are your goals, and reminding and going through again, are they important, why are they important." Providing feedback about performance was also observed to improve the participants' ability to set more realistic goals over time, and therapists observed participants' motivation for tackling future challenges and goals to be spurred by initial goal achievements.

Poor self-awareness and memory impairment reportedly affected clients' ability to recall goals, understand their problem areas, and participate in therapy. T2 stated, "He found it difficult to rate the importance or really sometimes even see why we were working on things, because of his memory," and she described the impact of depression on 1 participant's progress, stating that "amotivation and the symptoms of depression had such a big impact on those goals and his therapy in working towards those goals."

All the therapists expressed that goals evolved as circumstances changed or problems became clearer over time, and they noted the consequent need to "have the flexibility to adapt them" (T3) or to form new goals, which "needed to, I guess, be incorporated or included in what we were working on" (T2). The need to change goals or form new goals occurred in situations in which goal achievement came earlier than expected, in which a goal was too challenging, in which new problems emerged (such as depression), or the person lost interest in a goal area. T2 explained that her awareness of her client's problem with depression emerged as their therapeutic alliance developed, stating "That information was [of] the nature that it wouldn't come out at the start." On the whole, family members stated that the program goals set were appropriate to the participants' needs at that time (e.g., "At that stage, that was what was challenging him, I suppose" [SO of P8] and "I thought what he chose was good for him at that stage" [SO of P5]). Another SO stated, "You could see that probably they weren't the goals that needed to be set as time went on," although she also stated that "at the beginning they were very important, which is why they became the goals" (SO of P12).

A final theme related to the need to prioritize goals. $\mathrm{T} 1$, when reflecting on her therapy programs with the participants, felt that the focus was lost when multiple goals were worked on at one time. She stated, "Just picking the ones that were their absolute priority and really doing that thoroughly and doing it from the outset and getting a bit of an outcome there and then maybe picking up another couple" was preferable to working on goals in parallel. Focusing therapy on one priority goal at one time was also raised by the father of $\mathrm{P} 6$, who stated that "it would be a lot easier to focus on one goal at a time, and once that goal is achieved, work on the second goal, and so on." At the same time, this participant pointed out that the most important things should be worked on first. This family member defined "important goals" as skills that enabled independence, stating that "she's been doing a lot of cooking and things, but even without the cooking, you can always order McDonald's or pizza, you can still be independent, but you can't be independent if you can't have a shower on your own" (SO of P6).

\section{Discussion}

This study described the perspectives of participants with TBI, their significant others, and occupational therapists regarding their experience of a goal-directed, 12-week, individualized occupational therapy community rehabilitation program. The client-centered approach to goal planning resulted in most participants' generating their own goals; all three groups of participants emphasized the importance of clients' owning their goals. The participants expressed satisfaction with progress made and with the process of goaldirected therapy, although they identified some challenges. In particular, for 2 participants severe impairments of memory and self-awareness were reported as barriers to generating goals and attaining a sense of goal ownership. The link between impaired self-awareness after TBI and difficulties with setting realistic goals (Fleming \& Strong, 1995) and achieving rehabilitation outcomes (Fischer et al., 2004) has been previously reported.

In the current study, therapists and significant others emphasized the negative effect of impaired self-awareness, in some cases, on ability to identify problem areas. Goals were described as a tool, however, that can help increase selfawareness and participation over time as the client receives feedback about progress during engagement in goal-related activities.

Previous studies in this area have tended to focus on the goal-planning process and have reported self-awareness as a barrier to realistic goal planning, whereas this study highlights the value of providing support with setting goals, especially for clients with impaired self-awareness. Inability to identify program goals was not an exclusion criterion in this study, and in two cases a more time-consuming, therapistdirected approach was necessary to identify goals to enable initial engagement in therapy activities. The development of awareness of problems and more realism about targets over time was commonly described as a result of (1) rapport building, potentially enhanced within the context of a familiar environment in which the client may have felt 
more at ease to communicate; (2) provision of feedback from the therapist about performance; and (3) the experience of performing familiar tasks. Therefore, although impaired self-awareness may initially involve a lack of goal ownership, it may not always be a barrier to participation in goal-directed therapy in the context of community rehabilitation that includes family involvement and support in a collaborative approach to goal planning and rehabilitation.

As described in the literature (Fischer et al., 2004; Fleming \& Strong, 1995; Kuipers et al., 2004), some participants and their SOs identified the process of goal planning as challenging because of impaired self-awareness, reduced motivation, and cognitive impairments. The findings of this study emphasized that family members perceived a structured goal-planning process, in which the goal planning and therapy process is explained and facilitated, to be valuable for their relatives with TBI. Family members and participants felt that family involvement on the whole was positive for goal planning and that SOs should be included as partners in the process. Although participants and SOs interviewed in this study appeared to be a goal-orientated group (in that they expressed familiarity with the concept of goals), the literature about goals cautions that the concept of goals and changing oneself to attain something in the future (Kielhofner \& Barrett, 1997) is not a familiar concept to all, and a person's meaning and perspective on goals depends on his or her past experience.

The findings of this exploratory study indicate that once goals are set, they appear to have benefits for the therapy process. Those benefits are especially helpful in overcoming some of the common difficulties for people with TBI. First, representatives from the three groups identified the value of goals in providing structure; this benefit has previously been cited in terms of directing rehabilitation services and aiding in organization (Levack et al., 2006) and creating a process whereby regular review of performance in terms of goal achievement occurs (Kuipers et al., 2004; Levack et al., 2006). Participants interviewed in this study highlighted that having goals helped them feel organized and gave them a focus. Having a concrete plan to guide activities and help with planning future goals may be particularly valuable when a person has executive problems, such as poor planning skills. Therapists also highlighted the goal review process as valuable for structuring sessions and as a tool to motivate and focus patients at the start of therapy sessions.

This study identified the importance of a therapeutic alliance between therapists and clients that allows open communication and confrontation about difficult discussion topics; this type of alliance has previously been emphasized in the context of an outpatient clinic-based brain injury rehabilitation program (Schonberger, Humle, \& Teasdale,
2006). This situation may be enhanced in the community setting, where the relationship between therapist and client may be more of a partnership within the person's own home environment (Cott, 2004) and the therapist may be regarded as more of a friend than as an expert-teacher (Koch, Wottrich, \& Holmqvist, 1998). This open communication in a more relaxed atmosphere may encourage the provision of feedback to enhance self-awareness and support and facilitate clients in taking on new goals and challenges.

This study also supports previous research findings in that it demonstrated the value of involving significant others in identifying and planning client goals and increasing understanding of the client's context and problems (Kuipers et al., 2004). A notable finding of this study was that it identified common perspectives across the three groups, exclusive to therapists and SOs and to participants with TBI and SOs, but it identified no perspectives common to therapists and participants with TBI alone. This finding perhaps highlights the importance of SOs as mediators between therapists and clients in the process of goal planning and rehabilitation. This finding is especially relevant in a community context, where goal setting and rehabilitation increasingly involve the family (Seigert \& Taylor, 2004).

The identification of goals as tools to increase motivation was another finding that supports previous research (Gauggel $\&$ Hoop, 2004). Positive and regular feedback about concrete, task-specific performance and progress on meaningful goals was seen as a motivator for participants to strive, take on future challenges, and set new goals. The findings also support previous research highlighting that severe memory impairment can be a barrier to goal setting (Kuipers et al., 2004). However, a therapist in the current study was able to use goals to refocus and guide a client with severe memory impairment as to the purpose of the therapy sessions.

\section{Limitations and Future Research}

The generalizability of the findings may be limited because the sample size was small; it involved participants from one site; and the participants were involved in a specific, shortterm occupational therapy program. However, a strength of the study is the contribution of multiple perspectives in exploring goal-directed rehabilitation in the community setting for people with TBI. Moreover, simultaneous with their research intervention, participants were also undergoing a day hospital rehabilitation program, which did not offer the same structured, goal-planning process but may have influenced their views. During their interview, participants and SOs had the opportunity to compare and contrast their experiences. Although the findings were generally promising about the value of goal-directed therapy with people with 
TBI recently discharged from the hospital, further research is needed to establish its efficacy with this client group at various stages of rehabilitation. Particular attention to people with impaired self-awareness is warranted to examine the effects of therapist feedback and experiences of familiar task performance in familiar environments on a person's ability to set realistic goals.

\section{Conclusion}

In this study, which largely consisted of people with severe TBI and moderate to severe impairment in self-awareness, 10 of 12 patients were able to identify their own goals using the COPM, and goal ownership was a commonly identified theme in the interviews. The use of participants' goals to direct the content of the occupational therapy programs resulted in overall satisfaction with progress on goals. Although intrinsic factors, including poor motivation, impaired self-awareness, and cognitive impairment, were recognized as barriers to client-centered goal setting for people with TBI, we concluded that goals can be a valuable tool in rehabilitation by providing structure to overcome some of those difficulties. The study highlights the value of a therapist-facilitated, structured goal-planning process. $\mathrm{SO}$ are important mediators between clients and therapists to enhance the process of goal planning and rehabilitation in a community rehabilitation context.

\section{Acknowledgments}

We thank the participants, their significant others, and therapists for their participation. We wish to thank the Princess Alexandra Hospital Foundation and the Queensland Health Community Rehabilitation Research Scheme for providing funding to undertake this study. Emmah Doig was in receipt of a University of Queensland Postgraduate Scholarship while conducting this research.

\section{References}

Barnes, M. D., \& Ward, A. B. (2000). Textbook of rehabilitation medicine. Oxford, England: Oxford University Press.

Bodiam, C. (1999). The use of the COPM for the assessment of outcome on a neurorehabilitation unit. British Journal of Occupational Therapy, 62(3), 123-126.

Britten, N. (1995). Qualitative research: Qualitative interviews in medical research. British Medical Journal, 311, 251-253.

Cott, C. A. (2004). Client-centered rehabilitation: Client perspectives. Disability and Rehabilitation, 26(24), 1411-1422.

Doig, E. J., Fleming, J., \& Kuipers, P. (2008). Achieving optimal functional outcomes in community-based rehabilitation following acquired brain injury: A qualitative investigation of therapists' perspectives. British Journal of Occupational Therapy, 71(9), 360-370.

Eames, J., Ward, G., \& Siddons, L. (1999). Clinical audit of the outcome of individualised occupational therapy goals. British Journal of Occupational Therapy, 62(6), 257-260.

Fischer, S., Gauggel, S., \& Trexler, L. E. (2004). Awareness of activity limitations, goal setting, and rehabilitation outcome in patients with brain injuries. Brain Injury, 18(6), 547-562.

Fleming, J., \& Strong, J. (1995). Self-awareness of deficits following acquired brain injury. Considerations for rehabilitation. British Journal of Occupational Therapy, 58(2), 55-60.

Fleming, J., Strong, J., \& Ashton, R. (1996). Self-awareness of deficits in adults with traumatic brain injury: How best to measure? Brain Injury, 10, 1-15.

Gagne, D. E., \& Hoppes, S. (2003). Brief Report-The effects of collaborative goal-focused occupational therapy on selfcare skills: A pilot study. American Journal of Occupational Therapy, 57(2), 215-219.

Gauggel, S., \& Hoop, M. (2004). Goal-setting as a motivational technique for neurorehabilitation. In W. M. Cox \& E. Klinger (Eds.), Handbook of motivational counseling: Concepts, approaches and assessment (pp. 421-439). Chichester, England: Wiley.

Hamilton, B. B., Grainger, C. V., Sherwin, F. S., Zielezny, M., \& Tashman, J. S. (1987). Rehabilitation outcomes analysis and measurement. In M. J. Fuhrer (Ed.), A uniform data system for medical rehabilitation (pp. 137-147). Baltimore: Paul H. Brookes.

Jenkinson, N., Ownsworth, T., \& Shum, D. (2007). Utility of the Canadian Occupational Performance Measure in community-based brain injury rehabilitation. Brain Injury, 21(12), 1283-1294.

Joyce, B. M., Rockwood, K. J., \& Mate-Kole, C. C. (1994). Use of goal attainment scaling in brain injury in a rehabilitation hospital. American Journal of Physical Medicine and Rehabilitation, 73(1), 10-15.

Kielhofner, G., \& Barrett, L. (1997). Meaning and understanding in occupational forms: A study of therapeutic goal setting. American Journal of Occupational Therapy, 52(5), 345-353.

Kiresuk, T. J., \& Sherman, R. E. (1968). Goal attainment scaling: A general method for evaluating comprehensive community mental health programs. Community Mental Health Journal, 4, 443-453.

Koch, L. V., Wottrich, A. W., \& Holmqvist, W. L. (1998). Rehabilitation in the home versus the hospital: The importance of context. Disability and Rehabilitation, 20(10), 367-372.

Kuipers, P., Foster, M., Carlson, G., \& Moy, J. (2004). A preliminary exploration of goal setting in community based rehabilitation for people with brain impairment. Brain Impairment, 5(1), 30-41.

Lannin, N. (2003). Goal attainment scaling allows program evaluation of a home-based occupational therapy program. Occupational Therapy in Health Care, 17(1), 43-55.

Law, M., Baptiste, S., Carswell-Opzoomer, A., McColl, M. A., Polatajko, H., \& Pollock, N. (1991). The Canadian Occupational Performance Measure (COPM). Toronto: Canadian Association of Occupational Therapists. 
Lawler, J., Dowswell, G., Hearn, J., Forster, A., \& Young, J. (1999). Recovering from stroke: A qualitative investigation of the role of goal setting in late stroke recovery. Journal of Advanced Nursing, 30(2), 401-409.

Leach, E., Cornwell, P., Fleming, J., \& Haines, T. (in press). Patient-centered goal setting in a subacute rehabilitation setting. Disability and Rehabilitation.

Levack, W. M. M., Dean, S. G., McPherson, K. M., \& Siegert, R. J. (2006). How clinicians talk about the application of goal planning to rehabilitation for people with brain injuryVariable interpretations of value and purpose. Brain Injury, 20(13-14), 1439-1449.

MacLeod, G. M., \& MacLeod, L. (1996). Evaluation of client and staff satisfaction with a goal planning project implemented with people with spinal injuries. Spinal Cord, 34, 525-530.

Malec, J. F. (1999). Goal attainment scaling in rehabilitation. Neuropsychological Rehabilitation, 3/4(9), 253-275.

Mason, J. (2002). Qualitative researching (2nd ed.). London: Sage.

McAndrew, E., McDermott, S., Vitzakovitch, S., Warunek, M., \& Holm, M. B. (1999). Therapist and patient perceptions of the occupational therapy goal-setting process: A pilot study. Physical and Occupational Therapy in Geriatrics, 17(1), 55-63.

Ottenbacher, K. J., \& Cusick, A. (1989). Goal attainment scaling as a method of clinical service evaluation. American Journal of Occupational Therapy, 44(6), 519-525.

Paterson, B., \& Scott-Findlay, S. (2002). Critical issues in interviewing people with traumatic brain injury. Qualitative Health Research, 12(3), 399-409.

Patton, M. (2002). Qualitative research and evaluation methods (3rd ed.). Thousand Oaks, CA: Sage.

Pedretti, L. W. (1996). Occupational performance: A model for practice in physical dysfunction. In L. W. Pedretti (Ed.), Occupational therapy: Practice skills for physical dysfunction (4th ed., pp. 3-12). St Louis, MO: Mosby.

Phipps, S., \& Richardson, P. (2007). Occupational therapy outcomes for clients with traumatic brain injury and stroke using the Canadian Occupational Performance Measure. American Journal of Occupational Therapy, 61(3), 328-334.

Playford, E. D., Dawson, L., Limbert, V., Smith, M., Ward, C. D., \& Wells, R. (2000). Goal-setting in rehabilitation: Report of a workshop to explore professionals' perceptions of goal-setting. Clinical Rehabilitation, 14, 491-496.

Pollock, N. (1993). Client-centered assessment. American Journal of Occupational Therapy, 47(4), 298-301.

Rappaport, M., Hall, K. M., Hopkins, K., Belleza, T., \& Cope, D. N. (1982). Disability rating scale for severe head trauma: Coma to community. Archives of Physical and Medical Rehabilitation, 63(3), 118-123.

Reed, K., \& Sanderson, S. R. (1980). Concepts of occupational therapy. Baltimore: Williams \& Wilkins.
Ritchie, J., Spencer, L., \& O'Connor, W. (2003). Carrying out qualitative analysis. In J. Ritchie \& J. Lewis (Eds.), Qualitative research practice: A guide for social science students and researchers (pp. 219-262). Thousand Oaks, CA: Sage.

Schonberger, M., Humle, F., \& Teasdale, T. W. (2006). Subjective outcome of brain injury rehabilitation in relation to the therapeutic working alliance, client compliance, and awareness. Brain Injury, 20(12), 1271-1282.

Seigert, R. J., \& Taylor, W. J. (2004). Theoretical aspects of goal-setting and motivation in rehabilitation. Disability and Rehabilitation, 26(1), 1-8.

Sherer, M., Bergloff, P., Levin, E., High, W. M., Oden, K., \& Nick, T. G. (1998). Impaired awareness and employment outcome after traumatic brain injury. Journal of Head Trauma Rehabilitation, 13(5), 52-61.

Shut, H. A., \& Stam, H. J. (1994). Goals in rehabilitation teamwork. Disability and Rehabilitation, 16, 223-226.

Spencer, L., Ritchie, J., \& O'Connor, W. (2003). Analysis: Practices, principles, and processes. In J. Ritchie \& J. Lewis (Eds.), Qualitative research practice: A guide for social science students and researchers (pp. 199-218). Thousand Oaks, CA: Sage.

Sumison, T. (2004). Pursuing the client's goals really paid off. British Journal of Occupational Therapy, 67(1), 2-9.

Teasdale, G., \& Jennett, B. (1974). Assessment of coma and impaired consciousness. A practical scale. Lancet, 2, 81-84.

Toglia, J. P., \& Kirk, U. (2000). Understanding awareness deficits following brain injury. NeuroRehabilitation, 15(1), 57-70.

Trombly, C. A., Radomski, M. V., \& Davis, E. A. (1998). Achievement of self-identified goals by adults with traumatic brain injury: Phase I. American Journal of Occupational Therapy, 52(10), 810-818.

Trombly, C. A., Radomski, M. V., Trexel, C., \& Burnet-Smith, S. E. (2002). Occupational therapy and achievement of selfidentified goals by adults with acquired brain injury: Phase II. American Journal of Occupational Therapy, 56(5), 489-498.

Webb, P. M., \& Glueckauf, R. L. (1994). The effects of direct involvement in goal setting on rehabilitation outcome for persons with traumatic brain injuries. Rehabilitation Psychology, 39(3), 179-188.

Wilson, B. A., Evans, J. J., \& Keohane, C. (2002). Cognitive rehabilitation: A goal-planning approach. Journal of Head Trauma Rehabilitation, 17(6), 542-555.

Wressle, E., Eeg-Olofsson, A. M., Marcusson, J., \& Henriksson, C. (2002). Improved client participation in the rehabilitation process using a client-centered goal formulation structure. Journal of Rehabilitation Medicine, 34, 5-11.

Wressle, E., Oberg, B., \& Henriksson, C. (1999). The rehabilitation process for the geriatric stroke patient-An exploratory study of goal setting interventions. Disability and Rehabilitation, 21(2), 80-87. 